\title{
Diabetes mellitus, autonomia pessoal e práticas grupais: uma revisão integrativa
}

\author{
Diabetes mellitus, personal autonomy and group practices: an integrative review \\ Diabetes mellitus, autonomía personal y prácticas grupales: uma revisión integradora
}

Recebido: 04/01/2021 | Revisado: 04/01/2021 | Aceito: 07/01/2021 | Publicado: 08/01/2021

Richienne Thailane do Patrocínio Doval

ORCID: https://orcid.org/0000-0002-4679-4964

Universidade Federal do Rio Grande do Norte, Brasil E-mail: richienne97@gmail.com

Talita Araújo de Souza

ORCID: https://orcid.org/0000-0002-3138-6626 Universidade Federal do Rio Grande do Norte, Brasil E-mail: talitaaraujo23@hotmail.com

Marília Rute de Souto Medeiros

ORCID: https://orcid.org/0000-0003-1817-6859

Universidade Federal do Rio Grande do Norte, Brasil

E-mail: mariliarute@ hotmail.com

Ilisdayne Thallita Soares da Silva

ORCID: https://orcid.org/0000-0003-2421-8090

Universidade Federal do Rio Grande do Norte, Brasil E-mail: ilisdayne@yahoo.com.br

José Adailton da Silva

ORCID: https://orcid.org/0000-0002-6037-7649 Universidade Federal do Rio Grande do Norte, Brasil E-mail: silva.adailton@ufrn.edu.br

\begin{abstract}
Resumo
Objetivo: identificar evidências acerca das contribuições das práticas grupais na autonomia de pessoas que convivem com diabetes mellitus. Método: revisão integrativa do tipo qualitativa que incluiu artigos indexados nas bases de dados Literatura Latino Americana e do Caribe em Ciências da Saúde (LILACS), Medical Literature Analysis and Retrieval System Online (MEDLINE/PubMed), Scientific Electronic Library Online (SciELO.ORG) e Scopus (Elsevier), publicados entre os anos de 2015 a 2019, nas línguas portuguesa e inglesa. O período de coleta ocorreu entre os meses de junho a agosto de 2020. Resultados: incluíram-se 10 artigos, com metodologias variadas e realizados em países distintos. Apesar das variadas abordagens e metodologias, em todos os estudos foi demonstrado algum tipo de efetividade para a aquisição de autonomia pessoal e autocuidado através da participação em intervenção grupal. Conclusão: as práticas grupais desenvolvidas objetivando favorecer o aumento da autonomia e do autocuidado em pessoas convivendo com diabetes mellitus foram múltiplas e mostraram-se eficazes para serem utilizadas, sendo necessário se observar a necessidade de ocorrer reintervenções ao longo do tempo. $\mathrm{O}$ acompanhamento com o profissional enfermeiro foi apontado como fundamental no processo gradativo de aquisição destes e no uso das metodologias ativas que possuem baixo custo e, portanto, são cabíveis a serem reproduzidas em diferentes contextos. Palavras-chave: Autonomia pessoal; Processos grupais; Diabetes mellitus.
\end{abstract}

\begin{abstract}
Objective: identifying evidences of group practices contribution for autonomy of people who live with diabetes mellitus. Method: qualitative integrative review that includes indexed articles in these data bases: Literatura Latino Americana e do Caribe em Ciências da Saúde (LILACS), Medical Literature Analysis and Retrieval System Online (MEDLINE/PubMed), Scientific Electronic Library Online (SciELO.ORG) and Scopus (Elsevier). We used articles published between 2015 and 2019, in Portuguese and English. The period of collecting data occurred between June and August 2020. Results: we used ten articles with different methodologies performed in different countries. Despite the different approaches and methodologies, all the studies pointed out to some kind of effectiveness for personal autonomy and selfcare acquisition through participation in group intervention. Conclusion: there were several group practices developed to increase autonomy and selfcare on people who have diabetes mellitus and those practices turned out to efficient usage and new interventions were necessary throughout the time. The observance of a professional nurse was reported as essential for the gradual process of those practices acquisition and the usage of active low-cost methodologies, what turns them out to be reproduced in different contexts.
\end{abstract}

Keywords: Personal autonomy; Group process; Diabetes mellitus.

\section{Resumen}

Objetivo: identificar pruebas sobre las contribuciones de las prácticas grupales a la autonomía de las personas que viven con diabetes mellitus. Método: revisión integradora del tipo cualitativo que abarcó artículos indizados en las 
bases de datos Literatura Latinoamericana y del Caribe en Ciencias de la Salud (LILACS), Medical Literature Analysis and Retrieval System Online (MEDLINE/PubMed), Scientific Electronic Library Online (SciELO.ORG) y Scopus (Elsevier), publicadas entre 2015 y 2019, en los idiomas portugués e inglés. El período de recopilación fue de junio a agosto de 2020. Resultados: se incluyeron 10 artículos, con metodologías variadas y producidos en diferentes países. A pesar de la variedad de enfoques y metodologías, se demostró en todos los estudios algún tipo de eficacia para la adquisición de la autonomía personal y la autoatención mediante la participación en la intervención grupal. Conclusión: las prácticas grupales desarrolladas con el objetivo de favorecer el incremento de la autonomía y la autoatención de las personas que viven con diabetes mellitus fueron múltiples y se demostró que su utilización era efectiva, siendo necessário observar la necesidad de reintervenciones a lo largo del tiempo. Se señaló que el seguimiento con la enfermera profesional era crucial en el proceso gradual de su adquisición y en la utilización de metodologías activas de bajo costo y, por lo tanto, aptas para ser reproducidas en diferentes contextos.

Palabras clave: Autonomía personal; Procesos grupales; Diabetes mellitus.

\section{Introdução}

As Doenças Crônicas Não Transmissíveis (DCNT) se constituem como a principal causa de morte no mundo, havendo quatro que se destacam nesse quesito: doenças cardiovasculares, respiratórias crônicas, cânceres e, por fim, o diabetes mellitus (World Health Organization-WHO, 2018).

O diabetes mellitus (DM) é caracterizado como um distúrbio metabólico (Sociedade Brasileira de Diabetes-SBD, 2019) desencadeado pela falta ou ineficácia de um importante hormônio produzido no pâncreas, que age em diferentes tecidos e tem ação reguladora no metabolismo dos carboidratos, das proteínas e dos lipídeos. Esse hormônio é a insulina (SBD, 2017).

Embora seja somente um hormônio associado ao distúrbio, essa doença pode ser subdividida em vários tipos, sendo os mais conhecidos, os subtipos 1 e 2, além da DM gestacional (Federação Internacional de Diabetes-IDF, 2019). É válido ressaltar que em qualquer um dos casos a pessoa acometida pode vir a ter complicações ao longo da vida, como por exemplo, retinopatias, nefropatias, neuropatias periféricas, perda de membros e, no caso da DM gestacional, aumenta o risco de morte fetal. Sendo assim, independentemente do subtipo, pode haver uma diminuição na expectativa de vida do indivíduo acometido (WHO, 2016).

Com base nisso, o diagnóstico tardio ou o descuido ao tratar da doença pode gerar um aumento significativo de gastos que podem ser ou não quantificáveis. Cuidados médicos e incapacitações são quantificáveis, já a dor e a ansiedade não são quantificáveis, uma vez que envolve o impacto, enfrentamento e a convivência do indivíduo e do seu grupo familiar com a doença (SBD, 2017).

Mundialmente, há dados que apontam um expressivo aumento no número de adultos com DM. Segundo a Federação Internacional de Diabetes (IDF), em 2019, havia 463 milhões de pessoas diagnosticadas com a doença e estima-se que em 2045 esse número chegue a 700 milhões (IDF, 2019).

Tal aumento já vem sendo demonstrado há alguns anos. Em 1980, o Brasil ocupava a $7^{\text {a }}$ posição entre os dez países com maior número de adultos com diabetes (Zhou et al., 2016), e dados mais recentes indicam que o país passou a ocupar a $4^{\mathrm{a}}$ posição do ranking mundial (SBD, 2019; Zhou et al., 2016), estando atrás somente da Índia, da China e dos Estados Unidos da América (Zhou et al., 2016).

Por todas as informações referidas, atualmente esse agravo é considerado uma das maiores emergências de saúde global, já que ao longo dos anos se mostra como um crescente problema, no qual mais pessoas são acometidas e podem vir a desenvolver complicações (SBD, 2017), tendo sua qualidade de vida (QV) e autonomia pessoal prejudicada.

Para se entender melhor sobre, é necessário saber que a QV abrange variados meios da vida do indivíduo, como aspectos socioeconômicas, valores culturais e éticos, estado emocional ou o próprio estilo de vida (Oliveira, Antunes \& Oliveira, 2017). Já a autonomia possui múltiplos sentidos, podendo ser considerada como a capacidade de escolha, consciência de ações, ampliação de capacidades, direito de se expressar, oportunidade de dialogar, além da produção do seu próprio conhecimento (Freire, 2018; Portaria n 2.446, 2014). 
Considerando as informações expostas, é possível inferir que é necessária não somente a detecção precoce da doença, mas também a implantação de estratégias que possam auxiliar no controle do diabetes mellitus, além da construção da liberdade pelo sujeito, estando isso intimamente ligado à prática profissional (Freire, 2018) daqueles envolvidos no processo de cuidado, a fim de que o estar com uma doença crônica não seja necessariamente sinônimo de perda de autonomia.

Mediante a importância da discussão sobre a autonomia, que pode estar sujeita a implicações nas condições crônicas e tendo em vista a necessidade de preparo da equipe para intervir adequadamente sobre esta, definiu-se como objetivo deste estudo identificar as evidências acerca das contribuições das práticas grupais na autonomia de pessoas que convivem com diabetes mellitus.

\section{Metodologia}

Trata-se de uma revisão integrativa do tipo qualitativa, estruturada em seis etapas: 1) elaboração da questão de pesquisa; 2) eleição de bases de dados e os critérios de inclusão e exclusão; 3) seleção de estudos; 4) avaliação e coleta de informações; 5) interpretação e redação dos resultados; 6) apresentação da revisão/síntese do conhecimento (Cochrane Training, 2019).

A questão de pesquisa foi elaborada de acordo com o acrômio de População, Interesse e Contexto (PICo) (Lockwood, Munn \& Porritt, 2015). Considerou-se, assim, a seguinte estrutura: P - pessoas com diabetes; I - autonomia pessoal; Co práticas grupais. Dessa forma, elaborou-se a seguinte questão: qual a contribuição das práticas grupais na autonomia de pessoas convivendo com diabetes mellitus?

O levantamento bibliográfico foi realizado de junho a agosto de 2020, mediante acesso virtual às bases de dados: Literatura Latino Americana e do Caribe em Ciências da Saúde (LILACS), Medical Literature Analysis and Retrieval System Online (MEDLINE/PubMed), Scientific Electronic Library Online (SciELO.ORG) e Scopus (Elsevier).

Adotaram-se como critérios de inclusão: artigos com texto completo disponível em formato open acess online; dentro do intervalo de 2015 a 2019; que os resumos descrevessem práticas de educação em grupo às populações com diabetes; artigos em língua portuguesa e inglesa. Foram excluídos a literatura cinzenta (teses, dissertações, documento, relatos de caso, carta e livros), além dos demais tipos de revisões da literatura.

Para a busca nas bases de dados, foram selecionados descritores presentes nos Descritores em Ciências da Saúde (DeCS) e seus equivalentes no idioma inglês no Medical Subject Headings (MeSH). Neste caso, são eles: "Diabetes mellitus", “Autonomia Pessoal”, "Processos Grupais".

Para sistematizar a coleta da amostra, utilizou-se o formulário de busca avançada, respeitando peculiaridades e características distintas de cada base de dados. Os descritores foram combinados entre si com as expressões booleanas "AND” e "OR" dentro de cada termo da estratégia PICo, a fim de localizar os registros onde ocorressem os descritores selecionados previamente. A estratégia de busca nas bases de dados seguiu o protocolo conforme apresentado no Quadro 1.

Quadro 1 - Estratégia de busca com uso de descritores presentes no DeCS e MeSH. Santa Cruz, Rio Grande do Norte, Brasil, 2020 .

\begin{tabular}{|c|c|}
\hline Base de Dados & Estratégia de Busca \\
\hline Lilacs & Diabetes Mellitus (Palavras) and Autonomia Pessoal (Palavras) or Processos Grupais \\
\hline MEDLINE/PubMed & ((Diabetes Mellitus [Title/Abstract]) AND (Personal Autonomy [Title/Abstract])) OR \\
(Group Processes [Title/Abstract])
\end{tabular}


Conforme exposto acima, as buscas se deram nas bases de dados Lilacs, MEDLINE/PubMed, Scielo.ORG e Scopus utilizando as estratégias de busca conforme descrito na segunda coluna do quadro. Com isso, foram identificados 562 artigos atendendo os critérios elencados de elegibilidade, sendo 25 no LILACS, 72 no MEDLINE/PubMed, 378 SciELO.ORG, 87 na Scopus.

Para a seleção dos artigos, seguiram-se as recomendações do Preferred Reporting Items for Systematic Reviews and Meta-Analyses (PRISMA) (Liberati, A., 2009), conforme apresentado na Figura 1.

Figura 1 - Fluxograma de seleção dos estudos primários, elaborado a partir da recomendação PRISMA.

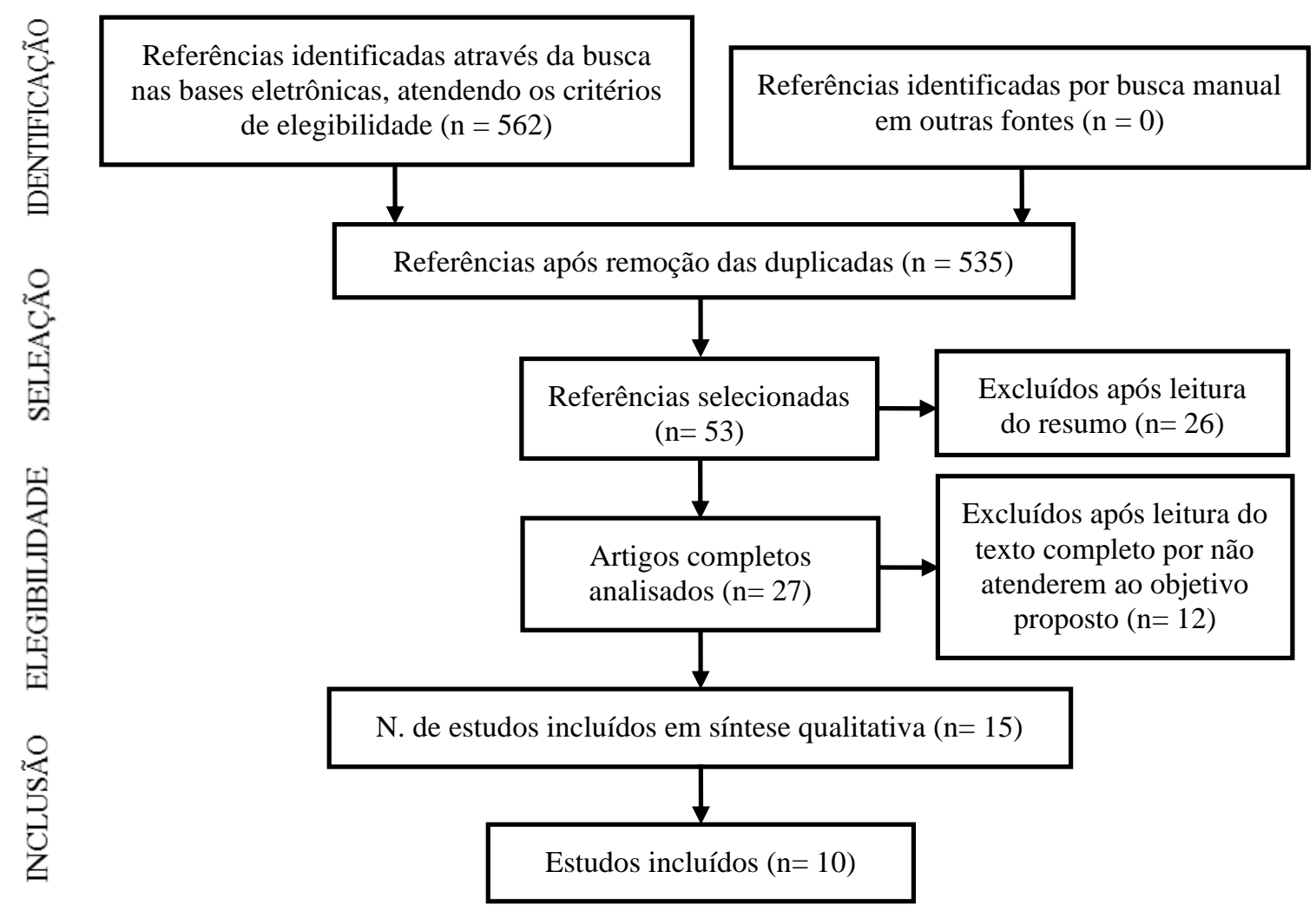

Fonte: (Liberati, A., 2009), com adaptação.

Realizando uma análise da figura acima, observa-se que 562 artigos atenderam os critérios de elegibilidade, havendo o descarte de 27 destes pela ocorrência de duplicidade. As exclusões observadas nas etapas demonstradas se deram pelas seguintes razões: apresentavam título e/ou resumo não aderente ao tema ou não respondiam ao objetivo do presente trabalho.

Dois pesquisadores independentes realizaram a seleção dos artigos, examinando cada um desses por título, resumo e texto completo. Para garantir a busca ampla, foram acessados por meio do portal de periódicos da Coordenação de Aperfeiçoamento de Pessoal de Nível Superior (CAPES), com reconhecimento na Universidade Federal do Rio Grande do Norte.

Após a seleção dos textos pelos pesquisadores, houve uma conciliação entre as concordâncias e discordâncias, com consulta detalhada aos textos completos. Dos 27 artigos selecionados, em 6 houve discordância na seleção entre os pesquisadores, resultando, por fim, na inclusão de 15 desses textos, perfazendo 10 artigos na revisão final.

Assim, por se tratar de um estudo feito a partir de dados já publicados, não se tratando de documentos que requeiram sigilo ético, foi desnecessária apreciação por Comitê de Ética em Pesquisa. 


\section{Resultados}

A amostra final foi composta por 10 artigos. Com relação às bases de dados nas quais os artigos foram selecionados, aquela que se destacou com maior quantitativo de artigos indexados conforme os critérios de inclusão foi a SciELO.ORG. Desta, cinco artigos compuseram a amostra por responderem ao questionamento do estudo. Os demais estavam indexados na Scopus (3), na LILACS (1) e na Medline/PubMed (1).

Foi identificada duplicidade em dois dos estudos elegíveis para compor a presente revisão. Um estava presente na LILACS e SciELO.ORG e, outro, presente na Medline/PubMed e Scopus. Desse modo, foi mantido apenas um de cada, sendo da LILACS e Medline/PubMed, respectivamente.

No Quadro 2 são apresentados os dados de forma descritiva, objetivando compreender o contexto das evidências disponíveis acerca das intervenções desenvolvidas voltadas à promoção da autonomia de pessoas convivendo com DM, favorecendo, desse modo, a reunião e organização do conhecimento sobre a temática investigada. Para isso, o perfil bibliográfico é representado por intermédio de dados, os quais incluem: título, autor, ano, país, objetivo, método e principais resultados.

Quadro 2 - Distribuição dos estudos selecionados, segundo variáveis de interesse. Santa Cruz, Rio Grande do Norte, Brasil, 2020.

\begin{tabular}{|c|c|c|c|}
\hline Título & $\begin{array}{l}\text { Autor/Ano/ } \\
\text { País } \\
\end{array}$ & Objetivo/Método & Principais resultados \\
\hline $\begin{array}{c}\text { Intervenção } \\
\text { educativa para a } \\
\text { promoção do } \\
\text { autocuidado de } \\
\text { idosos com } \\
\text { diabetes mellitus. }\end{array}$ & $\begin{array}{l}\text { Marques et al. } \\
\text { (2019). Brasil }\end{array}$ & $\begin{array}{l}\text { Avaliar a eficácia de uma } \\
\text { intervenção educativa de } \\
\text { enfermagem para o autocuidado. } \\
\text { Método: Quase-experimental do } \\
\text { tipo antes e depois }\end{array}$ & $\begin{array}{l}\text { Grupo intervenção }(\mathrm{GI})=\text { Melhora de aspectos } \\
\text { clínicos e nos quesitos avaliados pelo } \\
\text { Questionário de Autocuidado em Diabetes } \\
\text { (QAD). } \\
\text { Grupo Controle (GC) = Obteve melhora } \\
\text { clínica igualmente ao GI, exceto na média de } \\
\text { valores da pressão arterial sistólica/diastólica. }\end{array}$ \\
\hline $\begin{array}{l}\text { Literacy in health } \\
\text { and self-care in } \\
\text { people with type } 2 \\
\text { diabetes mellitus. }\end{array}$ & $\begin{array}{l}\text { Moura et al. } \\
\text { (2019). Brasil }\end{array}$ & $\begin{array}{l}\text { Avaliar o efeito de intervenções } \\
\text { educativas na adesão de } \\
\text { atividades de autocuidado e } \\
\text { letramento funcional em saúde. } \\
\text { Método: Quase-experimental }\end{array}$ & $\begin{array}{l}\text { Ocorreu melhora significativa do cuidado com } \\
\text { os pés, seguido de êxito nos resultados } \\
\text { referentes às atividades consideradas } \\
\text { indesejáveis relacionadas ao consumo } \\
\text { alimentar. Invariável para "tomar injeções de } \\
\text { insulinas conforme o recomendado". }\end{array}$ \\
\hline $\begin{array}{c}\text { Peer support to } \\
\text { improve diabetes } \\
\text { care: an } \\
\text { implementation } \\
\text { evaluation of the } \\
\text { Australasian Peers } \\
\text { for Progress } \\
\text { Diabetes Program. }\end{array}$ & $\begin{array}{l}\text { Aziz et al. } \\
\text { (2018). } \\
\text { Austrália }\end{array}$ & $\begin{array}{l}\text { Avaliar a implementação de um } \\
\text { ensaio clínico controlado } \\
\text { randomizado em grupo de um } \\
\text { programa de apoio de pares } \\
\text { baseado em grupo para melhorar } \\
\text { o autogerenciamento e controle } \\
\text { do diabetes tipo 2. } \\
\text { Método: Ensaio Clínico } \\
\text { Randomizado }\end{array}$ & $\begin{array}{l}\text { Maior parte dos usuários afirmou que o } \\
\text { programa os ajudou a controlar a diabetes no } \\
\text { seu cotidiano, ocorrendo afirmação da maioria } \\
\text { em pretender continuar a usar as estratégias } \\
\text { aprendidas, com maior percentual direcionado } \\
\text { para dieta saudável. } \\
\text { Motivos para falta de participação no } \\
\text { programa: problemas de saúde, falta de } \\
\text { tempo, compromissos de trabalho, local e } \\
\text { horário das reuniões, questões pessoais e } \\
\text { compromissos familiares. }\end{array}$ \\
\hline $\begin{array}{c}\text { Experiências, } \\
\text { necessidades e } \\
\text { expectativas de } \\
\text { pessoas com } \\
\text { diabetes mellitus. }\end{array}$ & $\begin{array}{l}\text { Silva, Amorim, } \\
\text { Valença \& } \\
\text { Souza } \\
\text { (2018a). Brasil }\end{array}$ & $\begin{array}{l}\text { Identificar as experiências, } \\
\text { necessidades e expectativas dos } \\
\text { sujeitos com diabetes, a partir da } \\
\text { perspectiva da autonomia para a } \\
\text { promoção da saúde. } \\
\text { Método: Estudo exploratório- } \\
\text { descritivo }\end{array}$ & $\begin{array}{l}\text { Estímulo para reflexão sobre ações e } \\
\text { acontecimentos diários, aquisição de novos } \\
\text { conhecimentos, a partir do compartilhamento } \\
\text { de experiências e das pautas apresentadas } \\
\text { pelos pesquisadores, conhecimento de } \\
\text { situações que causam insatisfação dos } \\
\text { usuários, autoconhecimento através da } \\
\text { percepção consciente daquilo que estava } \\
\text { dando certo ou não no controle diário do DM. }\end{array}$ \\
\hline $\begin{array}{c}\text { Diagnosis of } \\
\text { diabetes mellitus }\end{array}$ & $\begin{array}{c}\text { Silva et al. } \\
(2018 \mathrm{~b}) . \text { Brasil }\end{array}$ & $\begin{array}{l}\text { Compreender como os indivíduos } \\
\text { que vivem com diabetes lidam }\end{array}$ & $\begin{array}{lccc}\text { Geração de reflexões } & \text { a partir } & \text { do } \\
\text { compartilhamento } & \text { de } & \text { vivências } & \text { dos }\end{array}$ \\
\hline
\end{tabular}




\begin{tabular}{|c|c|c|c|}
\hline $\begin{array}{l}\text { and living with a } \\
\text { chronic condition: } \\
\text { participatory } \\
\text { study. }\end{array}$ & & $\begin{array}{l}\text { com o reconhecimento da } \\
\text { condição crônica em suas práticas } \\
\text { de cuidado à saúde. } \\
\text { Método: Pesquisa participativa }\end{array}$ & $\begin{array}{l}\text { participantes, diante do enfrentamento do } \\
\text { diagnóstico e da execução do autocuidado, } \\
\text { verbalização da insatisfação com o } \\
\text { adoecimento, valorização do processo de } \\
\text { aceitação e ressignificação da percepção de si } \\
\text { e de sua rede social, a fim de melhorar a } \\
\text { autonomia pessoal. }\end{array}$ \\
\hline $\begin{array}{l}\text { Intervenções } \\
\text { educativas para o } \\
\text { conhecimento da } \\
\text { doença, adesão ao } \\
\text { tratamento e } \\
\text { controle do } \\
\text { diabetes mellitus. }\end{array}$ & $\begin{array}{l}\text { Figueira, Boas, } \\
\text { Coelho, Freitas } \\
\& \text { Pace }(2017) \text {. } \\
\text { Brasil }\end{array}$ & $\begin{array}{l}\text { Avaliar o efeito de intervenções } \\
\text { educativas para conhecimento da } \\
\text { doença, adesão ao tratamento } \\
\text { medicamentoso e controle } \\
\text { glicêmico das pessoas com DM. } \\
\text { Método: Pesquisa de avaliação do } \\
\text { tipo antes e depois. }\end{array}$ & $\begin{array}{l}\text { Percebeu-se diminuição significativa dos } \\
\text { valores médios hemoglobina glicada (HbA1c); } \\
\text { aumento do score médio referente ao } \\
\text { conhecimento sobre o DM; e melhora na } \\
\text { média obtida na Medida de Adesão ao } \\
\text { Tratamento Medicamentoso no Diabetes } \\
\text { Mellitus - Antidiabéticos Orais (MAT - } \\
\text { ADOs). Não ocorreu melhora significante na } \\
\text { Medida de Adesão ao Tratamento } \\
\text { Medicamentoso no Diabetes Mellitus - } \\
\text { Insulinoterapia (MAT - Insulina). }\end{array}$ \\
\hline $\begin{array}{l}\text { Adesão e } \\
\text { empoderamento } \\
\text { de usuários com } \\
\text { diabetes mellitus } \\
\text { para práticas de } \\
\text { autocuidado: } \\
\text { ensaio clínico } \\
\text { randomizado. }\end{array}$ & $\begin{array}{l}\text { Macedo, Cortez, } \\
\text { Santos, Reis \& } \\
\text { Torres (2017). } \\
\text { Brasil }\end{array}$ & $\begin{array}{l}\text { Avaliar a adesão e } \mathrm{o} \\
\text { empoderamento do usuário por } \\
\text { meio da educação em grupo para } \\
\text { práticas de autocuidado e controle } \\
\text { glicêmico. } \\
\text { Método: Ensaio Clínico } \\
\text { Randomizado por cluster. }\end{array}$ & $\begin{array}{l}\text { GI obteve melhora significativa dos valores } \\
\text { médios da HbA1c e maior adesão de práticas } \\
\text { de autocuidado, enquanto que no GC não } \\
\text { houve alteração estatística significativa para } \\
\text { aspectos clínicos e do autocuidado. } \\
\text { Ambos obtiveram aumento da autoconfiança. }\end{array}$ \\
\hline $\begin{array}{l}\text { Relationship } \\
\text { between the users' } \\
\text { contact time in } \\
\text { educational } \\
\text { programs on } \\
\text { diabetes mellitus } \\
\text { and self-care skills } \\
\text { and knowledge. } \\
\end{array}$ & $\begin{array}{l}\text { Maia, Reis \& } \\
\text { Torres (2016). } \\
\quad \text { Brasil }\end{array}$ & $\begin{array}{l}\text { Verificar a relação entre o tempo } \\
\text { de contato de usuários em } \\
\text { programas educativos e as } \\
\text { variáveis conhecimento e e } \\
\text { autocuidado em DM. } \\
\text { Método: Longitudinal descritivo } \\
\text { de natureza quantitativa. }\end{array}$ & $\begin{array}{l}\text { Observou-se aumento médio nos escores tanto } \\
\text { para a variável conhecimento quanto para a } \\
\text { variável autocuidado; melhora do escore } \\
\text { médio no Questionário de Autocuidado para } \\
\text { DM2 (ESM) quando tempo de contato } \\
\text { profissional-usuário alcançou o período de } 8 \text { a } \\
14 \text { horas; e sem mudança detectada quando } \\
\text { inferior a } 8 \text { horas. }\end{array}$ \\
\hline $\begin{array}{l}\text { Effectiveness of } \\
\text { individual and } \\
\text { group } \\
\text { interventions for } \\
\text { people with type } 2 \\
\text { diabetes. }\end{array}$ & $\begin{array}{c}\text { Imazu, Faria, } \\
\text { Arruda, Sales \& } \\
\text { Marcon (2015). } \\
\text { Brasil }\end{array}$ & $\begin{array}{l}\text { Comparar a efetividade de duas } \\
\text { intervenções educativas usadas no } \\
\text { acompanhamento de indivíduo } \\
\text { com diagnóstico de DM2, no que } \\
\text { diz respeito ao conhecimento } \\
\text { sobre a doença, ao impacto na } \\
\text { qualidade de vida e à adoção de } \\
\text { ações de autocuidado. } \\
\text { Método: Estudo comparativo, } \\
\text { longitudinal, prospectivo. }\end{array}$ & $\begin{array}{l}\text { Os participantes da intervenção individual } \\
\text { tiveram resultados efetivos relacionados ao } \\
\text { impacto da doença na qualidade de vida, no } \\
\text { período de três e seis meses após a } \\
\text { intervenção. Apenas os participantes da } \\
\text { intervenção em grupo tiveram efeitos efetivos } \\
\text { em se tratando da adesão às práticas de } \\
\text { autocuidado. } \\
\text { Ao final de seis meses ocorreu redução } \\
\text { significativa somente para aqueles que } \\
\text { também participaram da intervenção em } \\
\text { grupo. }\end{array}$ \\
\hline $\begin{array}{l}\text { Group patient } \\
\text { education: } \\
\text { effectiveness of a } \\
\text { brief intervention } \\
\text { in people with } \\
\text { type } 2 \text { diabetes } \\
\text { mellitus in } \\
\text { primary health } \\
\text { care in Greece: a } \\
\text { clinically } \\
\text { controlled trial. }\end{array}$ & $\begin{array}{l}\text { Merakou, } \\
\text { Knithaki, } \\
\text { Karageorgos, } \\
\text { Theodoridise \& } \\
\text { Barbouni } \\
\text { (2015). Grécia }\end{array}$ & $\begin{array}{l}\text { Avaliar a eficácia da educação em } \\
\text { grupo com o uso de mapas de } \\
\text { conversação para pessoas com } \\
\text { DM tipo } 2 \text { em comparação com o } \\
\text { fornecimento de informações } \\
\text { padrão do médico da atenção } \\
\text { primária ao paciente. } \\
\text { Método: Ensaio clínico } \\
\text { controlado. }\end{array}$ & $\begin{array}{l}\text { GI = Após a intervenção, houve redução } \\
\text { significativa do Índice de Massa Corporal } \\
\text { (IMC), triglicerídeos, lipoproteína de baixa } \\
\text { densidade (LDL) e HbA1c. A lipoproteína de } \\
\text { alta densidade (HDL) permaneceu estável. } \\
\text { GC: Nenhuma mudança estatística com } \\
\text { relação ao IMC, mas obteve diminuição } \\
\text { significativa dos valores de HDL e LDL após } \\
6 \text { meses. }\end{array}$ \\
\hline
\end{tabular}


Nota-se, a partir da exploração do Quadro 2, que os artigos selecionados nesta revisão tiveram seu desenvolvimento e publicação em países distintos, sendo eles: Brasil (8), Austrália (1) e Grécia (1). Segundo o ano de publicação, obteve-se a seguinte distribuição: 2015 (2), 2016 (1), 2017 (2), 2018 (3) e 2019 (2).

As intervenções compiladas no Quadro 3 foram identificadas a partir da leitura das publicações nomeadas para o presente estudo e tiveram a realização distinta em alguns aspectos quanto ao tempo, à técnica utilizada, às características da população e ao objetivo, mas mantiveram os comportamentos de autonomia e autocuidado como eixo principal (alimentação saudável, exercícios físicos, adesão à medicação, automonitorização da glicemia e/ou cuidados com os pés).

Quadro 3 - Distribuição das intervenções/metodologias utilizadas combinadas ou não nos estudos selecionados. Santa Cruz, Rio Grande do Norte, Brasil, 2020.

\begin{tabular}{|l|r|}
\hline \multicolumn{1}{|c|}{ Metodologia/Intervenção } & Número de vezes usadas \\
\hline Acompanhamento convencional + Ligação telefônica & 1 \\
\hline Práticas grupais + Ligação telefônica & 3 \\
\hline Preenchimento de instrumentos & 8 \\
\hline Acompanhamento convencional (consultas de rotina) & 2 \\
\hline Práticas grupais + Sem aplicação de ligação telefônica & 4 \\
\hline Práticas grupais + Visita domiciliar & 1 \\
\hline
\end{tabular}

Fonte: Autores (2020).

Constata-se que preenchimento de algum tipo de instrumento obteve a maior ocorrência como forma de avaliar a eficácia das intervenções. A ligação telefônica foi utilizada como um meio conciliador em três casos estudados, mas ainda houve maior ocorrência de práticas que não utilizaram esse recurso.

Para mensurar os resultados das intervenções, em oito dos 10 artigos houve a utilização de algum tipo de medidor de mudança de comportamento e autocuidado. Os mais utilizados foram o QAD - Questionário de Autocuidado em Diabetes (Marques et al., 2019; Moura et al., 2019; Imazu et al., 2015) e o DKN-A - Questionário de conhecimento do Diabetes (Figueira et al., 2017; Maia et al., 2016; Imazu et al. 2015). Além desses, ainda houve a utilização do PAID - Questionário de Impacto na Qualidade de Vida em Diabetes (Imazu et al., 2015), ESM e DES-SF - Questionário de empoderamento para o DM2 (Macedo et al., 2017), MAT - ADOs e MAT - Insulina (Figueira et al., 2017). Apenas um fez uso de um instrumento elaborado, buscando avaliar a satisfação dos usuários com o grupo e as intervenções realizadas (Aziz et al., 2018).

Cabe mencionar que, apesar do objetivo do presente trabalho ser inerente à autonomia pessoal, observou-se que os estudos trazem de forma abrangente o autocuidado.

\section{Discussão}

Conforme exposto anteriormente, o autocuidado foi amplamente abordado nos resultados encontrados, sendo assim, incube abordar sobre tal fator. A partir do estudo da teoria de Dorothea Orem, tem-se como definição deste o comportamento aprendido e realizado pelas pessoas para si mesmas a partir de um pensamento crítico e reflexivo de situações com o intuito de proporcionar um benefício na sua saúde, vida e bem-estar (Bezerra et al., 2018).

Depreende-se, dessa forma, que a autonomia pessoal desenvolvida por meio da ampliação de capacidades e escolhas atua potencializando ações de autocuidado do sujeito. Assim, essas duas categorias estão vinculadas, uma vez que os sujeitos podem explorar e conhecer sobre algo e, a partir disso, escolher aplicar ou não na sua realidade para benefício próprio (Oliveira, Carvalho, Gondim, \& Freire, 2020). Sendo assim, a melhora do autocuidado está diretamente relacionada à aquisição de autonomia. 
Apesar da relevância e dos benefícios das intervenções educacionais para o controle de doenças, pelo fato destas estimularem a adoção de comportamentos de forma voluntária, terem como foco o sujeito, a problematização, a valorização de experiências e realidades, e, portanto, terem capacidade de reorientar práticas dentro do contexto do conhecimento sobre o processo saúde-doença-adoecimento (Alves \& Aerts, 2011), os resultados demonstram que ainda há poucos estudos desenvolvidos nos últimos anos acerca de intervenção com grupos educativos voltados para a autonomia pessoal.

Em todos os trabalhos analisados, seja com abordagem quantitativa ou qualitativa, houve evidência que a prática educativa em grupo é capaz de induzir mudanças de comportamento e/ou ajudar na melhoria do controle metabólico (Marques et al., 2019; Moura et al., 2019; Aziz et al., 2018; Silva et al., 2018a; Silva et al., 2018b; Figueira et al., 2017; Macedo et al., 2017; Maia et al., 2016; Imazu et al., 2015; Merakou et al., 2015), sendo válido ressaltar que esse efeito positivo pode ter uma duração limitada, como demonstrado no estudo de Imazu (2015), que ao final de seis meses apresentou uma redução significativa dos escores para o autocuidado de indivíduos que participaram da intervenção grupal.

Esse achado converge com o que é conhecido como educação em saúde, já que esta é considerada com uma prática de constante criação de conhecimento, ocorrendo de forma gradativa e interativa (Ribeiro, 2018), necessitando se pensar na viabilidade de ocorrer reintervenções e monitorização periódica dos usuários.

Diante disso, relembra-se uma importante dimensão da Atenção Primária à Saúde (APS): a criação de vínculo entre pessoas/famílias/comunidade a profissionais/equipes de saúde e a longitudinalidade de ações, objetivando a continuidade do cuidado e da integralidade da assistência (Portaria no 2.436/2017), proporcionando ao usuário maior satisfação nas ações em que é inserido. Isso já foi explicitado em outros estudos de revisão, como dos autores Aguayo-Verdugo et al. (2019) e Cardoso et al. (2015), em que o fator supracitado se mostra como determinante para que os usuários se sintam acolhidos e comprometidos com as ações a partir da criação de confiança entre os envolvidos.

Retomando ao componente "tempo", Maia (2016) aponta que para ocorrer um efeito positivo na variável autocuidado são necessárias pelo menos oito horas de contato entre profissional-usuário. Esse achado é importante ser mais aprofundado em outros estudos, uma vez que nessa revisão há registro de uma intervenção grupal pontual que obteve resultado positivo com relação tanto aos aspectos clínicos quanto aos analisados através do instrumento QAD (Marques et al., 2019).

Em relação às intervenções, destacam-se as características que possuem em comum. Em seis dos dez artigos há menção da participação do profissional enfermeiro durante a realização das intervenções, tendo isso ocorrido pessoalmente ou por contato telefônico (Marques et al., 2019; Moura et al., 2019; Macedo et al., 2017; Figueira et al., 2017; Maia et al., 2016; Imazu et al., 2015). Em sete, o componente psicológico contou como parte importante das intervenções por meio de diálogo motivacional, mudança de comportamento ou resolução de problemas (Aziz et al., 2018; Silva et al., 2018a; Silva et al., 2018b; Macedo et al., 2017; Figueira et al., 2017; Maia et al., 2016; Imazu et al., 2015). Em quatro houve a alocação de indivíduos para se montar grupos diferentes, a fim de comparar os resultados. Sendo assim, havia aqueles que participaram do grupo intervenção e aqueles do grupo controle (Marques et al., 2019; Macedo et al., 2017; Merakou et al., 2015; Imazu et al., 2015).

Com relação aos estudos que utilizaram esse último tipo de seguimento para avaliar a efetividade das ações, percebeuse que além de melhorias relacionadas às ações voltadas para autocuidado, participantes das intervenções em grupo também puderam obter melhoras em aspectos clínicos (Marques et al., 2019; Macedo et al., 2017; Merakou et al., 2015).

A adesão dos usuários para tomar as injeções de insulina conforme prescrição não sofreu variação significante estatisticamente em nenhum dos estudos. Foram três trabalhos que abordaram sobre esse quesito, e eles tiveram tempos de execução diferentes, sendo assim, mostrou-se que o tempo não é necessariamente quem influência para adoção desse hábito (Marques et al., 2019; Moura et al, 2019; Figueira et al., 2017).

Considerando os achados referentes à atuação do enfermeiro nas ações e nas peculiaridades que permeiam a convivência de uma pessoa com o DM, ressalta-se a importância de existir um olhar mais aguçado desse profissional, com 
intuito de melhorar as práticas de autocuidado mediante o estímulo do exercício de sua autonomia pessoal, na perspectiva de uma atuação multidimensional e qualificada, promovendo atividades que estimulem a participação dos usuários, abnegando o modelo curativista de assistência à saúde (Bomfim et al., 2017).

Dentre os estudos, destacam-se três (Macedo et al., 2017; Marques et al., 2019; Figueira et al., 2017), por demonstrarem em seus resultados melhorias estatisticamente significativas tanto para as práticas de autocuidado como também para o aspecto clínico, nesse caso, especialmente para o índice da hemoglobina glicada. Com isso, é pertinente destacar aspectos que ambas possuem em comum. Todas tiveram um período de aplicação de intervenções superior a seis meses e tiveram o profissional enfermeiro a frente para conduzir os momentos. Quanto às intervenções, Macedo (2017) e Marques (2019) adotaram as práticas grupais conciliadas à ligação telefônica no intervalo entre os ciclos de encontros. Já Figueira (2017) não conduziu as intervenções aliando ao contato telefônico.

Além das intervenções já mencionadas utilizando algum instrumento capaz de medir estatisticamente as variações nos participantes antes e após o período de práticas grupais, há dois estudos (Silva et al., 2018a, 2018b) que trazem a roda de conversa como intervenção usada, o que é importante para se ter a conversação diante de um espaço que viabiliza aos sujeitos a troca e o desenvolvimento de saberes, empoderamento pessoal e, consequentemente, maior autonomia (Adamy, Zocche, Vendruscolo, Santos \& Almeida, 2018).

É possível inferir que estudos com esse tipo de abordagem não se caracterizam como inferiores aos demais, já que Silva (2018a, 2018b) trouxe em seus resultados quesitos importantes para indivíduos conquistarem sua autonomia a partir da identificação de elementos que contribuem para mudanças no estilo de vida, da compreensão de situações envolvendo a aceitação e o enfrentamento através da reflexão, do esclarecimento de dúvidas, da aquisição de novos conhecimentos, da oportunidade de verbalização sobre a insatisfação e da aceitação com a doença, além da identificação de situações que causam desconforto nos participantes.

Pondo fim as análises, a linguagem incompreensível é apontada no estudo de Silva (2018b) como um fator causal para a insatisfação do usuário. Isso oportuniza a se pensar que, além de estímulo para se participar de atividades que promova ao indivíduo conhecimento das práticas de autocuidado correlacionada à autonomia pessoal, existe também a necessidade de oportunizar que estes tenham também um domínio na estratégia de Letramento Funcional em Saúde (LFS), a fim de que este tenha acesso a informações em diferentes contextos e se sinta estimulado a buscar os serviços de saúde, de modo que a comunicação seja efetiva entre profissionais e usuários (Moura et al, 2019).

\section{Conclusão}

Esta revisão permitiu que se fosse conhecida uma variedade de intervenções aplicadas em práticas educativas em grupo, em diferentes localidades, com o intuito de aumentar a autonomia pessoal de pessoas convivendo com DM. Isso oportunizou que novos saberes fossem construídos a partir da participação ativa dos usuários nessas situações, facilitando a aprendizagem de forma ativa e promovendo a ressignificação da condição que se está inserido.

Acerca das intervenções, os resultados encontrados sugerem que as práticas grupais são efetivas na perspectiva da autonomia pessoal de pessoas convivendo com DM, uma vez que em diferentes estudos ocorreu o aumento do conhecimento sobre a doença, o empoderamento, a adesão das práticas de autocuidado medidas por intermédio de instrumentos específicos, o autoconhecimento por meio da reflexão e compartilhamento de experiências, além da melhora do índice glicêmico evidenciada pelos resultados da hemoglobina glicada.

Importante mencionar que as intervenções grupais citadas, nas quais se faz uso de rodas de conversa, mapas de conversação, uso de cartazes, fontes áudio-visuais, acompanhamento telefônico e visitas domiciliares como conciliadoras, são de baixo custo, necessitando, primordialmente, da disponibilidade da equipe, da organização de recursos e metodologias e do 
interesse do sujeito, devendo ainda, ocorrer a análise da ocorrência de reintervenções afim de que a longitudinalidade das ações ocorra, bem como a continuidade do cuidado, entendendo-se a relevância da educação permanente em saúde.

Por conseguinte, a presença do enfermeiro como facilitador do processo educativo foi identificada, em seis, entre os oito estudos encontrados. A educação em saúde está inserida no contexto da atuação da enfermagem, como meio para o estabelecimento de uma relação dialógico-reflexiva entre enfermeiro e cliente, devendo este, conscientizar-se sobre sua situação de saúde-doença, e perceber-se como sujeito de transformação de sua própria vida. Portanto, por se constituir uma peça fundamental nesse meio, é necessário que estes sejam qualificados para atuar de forma a ter uma prática que integre os usuários, mas que, ainda assim, seja levada em consideração a necessidade de haver a centralidade na pessoa que participa e espera ser compreendida.

Nesse sentido, é imprescindível que ocorra mais estudos envolvendo a presente temática com um enfoque ao tempo em que o indivíduo é exposto as intervenções e o seu contato direto com os profissionais envolvidos, afim de que além de informações sobre intervenções e metodologias grupais efetivas, se tenha também uma caracterização real sobre o período de realização eficaz, afim de que para estratégias sejam pensadas e implantadas nos serviços de saúde.

\section{Referências}

Adamy, E. K., Zocche, D. A. A., Vendruscolo, C., Santos, J. L. G., \& Almeida, M. A. (2018). Validation in grounded theory: conversation circles as a methodological strategy. Revista Brasileira de Enfermagem, 71(6), 3121-3126. https://doi.org/10.1590/0034-7167-2017-0488.

Alves, G. G., \& Aerts, D. (2011). As práticas educativas em saúde e a Estratégia Saúde da Família. Ciência \& Saúde Coletiva, 16(1):319-25. http://dx.doi.org/10.1590/S14 13-81232011000100034.

Verdugo, A., Valeska, N., Yañez, O., \& Ester, A. (2019). Intervenções de enfermagem em pacientes com diabetes mellitus tipo 2 que aumentam seus comportamentos de autocuidado: revisão sistemática. Enfermería Actual de Costa Rica, (36), 116-129. https://dx.doi.org/10.15517/revenf.v0i36.34238.

Aziz, Z., Riddell, M. A., Absetz, P., Brand, M., Oldenburg, B., Dunbar, J. A., \& Zaini, A. (2018). Peer support to improve diabetes care: an implementation evaluation of the Australasian Peers for Progress Diabetes Program. BMC Public Health, 18, 262. https://doi.org/10.1186/s12889-018-5148-8.

Bezerra, M. L. R., Faria, R. P. R., Jesus, C. A. C., Reis, P. E. D., Pinho, D. L. M., \& Kamada, I. (2018). Aplicabilidade da teoria do déficit do autocuidado de Orem no Brasil: uma revisão integrativa. J Manag Prim Health Care. https://doi.org/10.14295/jmphc.v9i0.538.

Bomfim, E. S., Araújo, I. B., Santos, A. G. B., Silva, A. P., Vilela, A. B. A., \& Yarid, S. D. (2017). Nurse activity on educational practices in the family health strategy. Revista de enfermagem UFPE on line. 1398-402. 10.5205/reuol.10263-91568-1- RV.1103sup201711.

Cardoso, A. F., Queirós, P., \& Ribeiro, C. F. (2015). Intervenções para a aquisição do autocuidado terapêutico da pessoa com diabetes mellitus: revisão sistemática de literatura. Revista Portuguesa de Saúde Pública. https://doi.org/10.1016/j.rpsp.2015.04.001.

Federação Internacional de Diabetes (IDF). (2019). Atlas de Diabetes da IDF. Bélgica.

Figueira, A. L. G., Boas, L. C. G. V., Coelho, A. C. M., Freitas, M. C. F., \& Pace, A. E. (2017). Intervenções educativas para o conhecimento da doença, adesão ao tratamento e controle do diabetes mellitus. Revista Latino-Americana de Enfermagem. 10.1590/1518-8345.1648.2863.

Freire, P. (2018). Pedagogia da autonomia: Saberes necessários à prática educativa. (56a ed.), Paz e Terra.

Higgins, J. P. T., Thomas, J., Chandler, J., Cumpston, M., Li, T., Page, M. J., \& Welch, V. A. (2019). Cochrane Handbook for Systematic Reviews of Interventions version 6.0 .

Imazu, M. F. M., Faria, B. N., Arruda, G. O., Sales, C. A., \& Marcon, S. S. (2015). Effectiveness of individual and group interventions for people with type 2 diabetes. Revista Latino-Americana de Enfermagem, 23 (2), 200-207. https://doi.org/10.1590/0104-1169.0247.2543.

Liberati, A., Altman, D. G., Tetzlaff, J., Mulrow, C., Gøtzsche, P. C., Loannidis, J. P. A., \& Moher, D. (2009). The PRISMA Statement for Reporting Systematic Reviews and Meta-Analyses of Studies That Evaluate Health Care Interventions: Explanation and Elaboration. PLoS Med, 6 (7). 10.1371/journal.pmed.1000100.

Lockwood, C., Munn, Z., \& Porritt, K. (2015). Qualitative research synthesis methodological guidance for systematic reviewers utilizing meta-aggregation. International Journal of Evidence-Based Healthcare, 13(3), 179-187. 10.1097/XEB.0000000000000062.

Macedo, M. M. L., Cortez, D. N., Santos, J. C., Reis, I. A., \& Torres, H. C. (2017). Adesão e empoderamento de usuários com diabetes mellitus para práticas de autocuidado: ensaio clínico randomizado. Revista da Escola de Enfermagem da USP. https://doi.org/10.1590/s1980-220x2016050303278.

Maia, M. A., Reis, I. A., \& Torres, H. C. (2016). Relationship between the users' contact time in educational programs on diabetes mellitus and self-care skills and knowledge. Revista da Escola de Enfermagem da USP, 50(1):59-64. http://dx.doi.org/10.1590/S0080-623420160000100008.

Marques, M. B., Coutinho, J. F. V., Martins, M. C., Lopes, M. V. O., Maia, J. C. \& Silva, M. J. (2019). Intervenção educativa para a promoção do autocuidado de idosos com diabetes mellitus. Revista da Escola de Enfermagem da USP. https://doi.org/10.1590/s1980-220x2018026703517. 
Merakou, K., Knithaki, A., Karageorgos, G., Theodoridis, D., \& Barbouni, A. (2015). Group patient education: effectiveness of a brief intervention in people with type 2 diabetes mellitus in primary health care in Greece: a clinically controlled trial. Health Education Research, 30 (2). https://doi.org/10.1093/her/cyv001.

Moura, N. S., Lopes, B. B., Teixeira, J. J. D., Oriá, M. O. B., Vieira, N. F. C. \& Guedes, M. V. C. (2019). Literacy in health and self-care in people with type 2 diabetes mellitus. Revista Brasileira de Enfermagem, 72(3), 700-706. https://doi.org/10.1590/0034-7167-2018-0291.

Oliveira, D. V., Antunes, M. D., \& Oliveira, J. F. (2017). Ansiedade e sua relação com a qualidade de vida em idosos: revisão narrativa. Cinergis, 18(4), 316322. http://dx.doi.org/10.17058/cinergis.v18i4.9951.

Oliveira, G. S., Carvalho, M. F. A. A., Gondim, L. S. S., \& Freire, A. K. S. (2020). Self-care and its consequences in health production of health in psychosocial care. Research Society and Development. 10.33448/rsd-v9i7.4942.

Portaria $n^{\circ}$ 2.446, de 11 de novembro de 2014. (2014). Redefine a Política Nacional de Promoção da Saúde (PNPS).

Portaria $n^{\circ}$ 2.436, de 21 de setembro de 2017. (2017). Política Nacional de Atenção Básica (PNAB).

Ribeiro, K. G., Andrade, L. O. M., Aguiar, J. B., Moreira, A. E. M. M., \& Frota, A. M. (2018). Educação e saúde em uma região em situação de vulnerabilidade social: avanços e desafios para as políticas públicas. Interface-Comunicação, Saúde, Educação. 22, 1387-1398. https://doi.org/10.1590/180757622017.0419 .

Silva, J. A., Amorim, K. P. C., Valença, C. N., \& Souza, E. C. F. (2018a). Experiências, necessidades e expectativas de pessoas com diabetes mellitus. Revista Bioética. 26 (1), 95-101. https://doi.org/10.1590/1983-80422018261230.

Silva J. A. D., Souza E. C. F., Böschemeier, A. G. E., Costa C. C. M., Bezerra H. S. \& Feitosa E. E. L. C. (2018b). Diagnosis of diabetes mellitus and living with a chronic condition: participatory study. BMC Public Health, 5;18(1):699. 10.1186/s12889-018-5637-9.

Sociedade Brasileira de Diabetes (SBD). (2017). Diretrizes da Sociedade Brasileira de Diabetes 2017-2018.

Sociedade Brasileira de Diabetes (SBD). (2019). Conduta terapêutica no diabetes tipo 2: Algoritmo SBD 2019.

World Health Organization (WHO). (2018). Noncommunicable diseases in the Region of the Americas: facts and figures. Geneva.

World Health Organization (WHO). (2016). Global report on diabetes 2016. Geneva.

Zhou, B., Lu, Y., Hajifathalian, K., Bentham, J., Di Cesare, M., Danaei, G., \& Bennett, J. (2016). Worldwide trends in diabetes since 1980: a pooled analysis of 751 population-based studies with 4.4 million participants. The Lancet. 387(10027), 1513-1530. Elsevier BV. https://doi.org/10.1016/S01406736(16)00618-8. 